\title{
Simplified V-Shape Incision Technique for Saving Hair Follicles
}

In Sung Yeo, Seung Jun Shin, Yu Jin Jang, Myong Chul Park

Department of Plastic and Reconstructive Surgery, Ajou University Hospital, Suwon, Korea
No potential conflict of interest relevant to this article was reported.
Background The scalp is the second most visible part of the anatomy next to the face. Thus, aesthetic considerations such as scars and alopecia are important when planning excision of a mass from the scalp. Aesthetic results have not been satisfactory due to scar widening and hair follicle injury. Many methods have been tried to improve these results, however, they require multiple procedures and longer operation time. Methods We evaluated a easy and time-saving V-shape incision for excising a scalp mass. This technique of simplified $\mathrm{V}$-shape incision was performed in 32 patients with benign scalp masses treated from September 2011 to March 2013. The incisions were performed with a blade to make a V-shape margin and preserve deep hair follicles at the flap margins. The defect was closed by approximation of two edges.

Results All tumors were benign and superficial. The size of the scalp masses was 0.4$2.0 \mathrm{~cm}$ in width and $1.0-5.0 \mathrm{~cm}$ in length. Many hairs from the deep hair follicles grew into the scar, making the scar line almost unnoticeable in all 32 patients. Although some scar widening was evident, the number of hairs per unit was high enough to camouflage the scars.

Conclusions The simplified V-shape incision was beneficial as a simple, mini-surgical, time-saving procedure. Although the scars were slightly wider, they were covered with penetrating hairs and became unnoticeable.

Keywords Hair follicle, Scalp, Cicatrix, Alopecia

\section{INTRODUCTION}

Cicatrical alopecia is considered inevitable following scalp operations. The wound area on the scalp appears hairless even with optimal wound conditions. Aesthetic results have not been satisfactory because of scar widening and injury to hair follicles.

Many methods have been attempted to improve results. Examples include wedge excision and double relaxation suture, trichophytic closure, and the single scar technique $[1,2]$. While these

Received: Mar 28, 2014 Revised: May 25, 2014 Accepted: May 27, 2014 Correspondence: Myong Chul Park Department of Plastic and Reconstructive Surgery, Ajou University Hospital, 164 Worldcup-ro, Yeongtong-gu, Suwon 443-380, Korea. E-mail: mpark@ajou.ac.kr

Copyright @ 2014 The Korean Society for Aesthetic Plastic Surgery.

This is an Open Access article distributed under the terms of the Creative Commons Attribution Non-Commercial License (http://creativecommons.org/licenses/by-nc/3.0/) which permits unrestricted non-commercial use, distribution, and reproduction in any medium, provided the original work is properly cited. www.e-aaps.org methods have achieved good results to effectively minimize scars and camouflage donor scars, they require multiple procedures, longer operation time, and longer learning curves.

Herein, we introduce an effective method of a simplified V-shape incision on the scalp and report good results.

\section{METHODS}

Thirty-two patients (20 males and 12 females; mean age, 23.4 years; range, 1-63 years) who visited our hospital from September 2011 to March 2013 and were treated to remove a scalp tumor were selected. The size of the mass was measured before each surgical procedure. After removing the tumors, the specimens were sent to the department of pathology for confirmative diagnosis. Clinical photos were taken before and after each operation. Patients were followed-up for 6 months.

The procedure was performed with a field block using $1 \%$ lidocaine with a 1:100,000 dilution of epinephrine in the subcutane- 
ous and dermal planes. Each incision was simply performed with a blade. The bevel angle was $50-70^{\circ}$ horizontal to the skin surface, preserving the many deep hair follicles at both margins (Fig. 1 and 2B). After complete excision of the mass, a scalp laxity examination was conducted to assess the safety of tension-free closure of the defect. The scalp laxity test is important to determine scalp flexibility. Scalp flexibility is determined by how much the skin can stretch and how much the upper layers of the skin can glide over the bottom layers. If there was moderate tension, minimal dissection was done at the supra galea level to release the tension, and coverage of the defect was simply achieved by approximating the two edges. The relaxation suture was performed minimally with absorbable material (3-0 or 4-0 Vicryl [Ethicon, NJ, USA] or PDS [Ethicon, NJ, USA]) in a simple interrupted manner. The subcutaneous and dermal layers were closed with absorbable material (3-0 or 4-0 Vicryl or PDS) in a simple interrupted manner.
The superficial skin layer was closed with non-absorbable material (4-0 or 5-0 Prolene [Ethicon, NJ, USA]) avoiding injury to the superficial hair follicles in a simple interrupted manner. Surgical sutures were usually removed 10-13 days after the operation.

\section{RESULTS}

All tumors were benign superficial tumors (epidermoid cyst, seborrheic keratosis, pilomaticoma, nevus sebaceous, intradermal nevus, compound nevus, blue nevus, etc.). The size of the scalp masses was $0.4-2.0 \mathrm{~cm}$ in width and $1.0-5.0 \mathrm{~cm}$ in length (Table 1). The depth of incision to complete removal was the subcutaneous level. Three patients had moderate tension on the scalp laxity test, so undermining procedures at the supra galea level were performed to release tension. Approximation and skin closure were performed easily without tension.

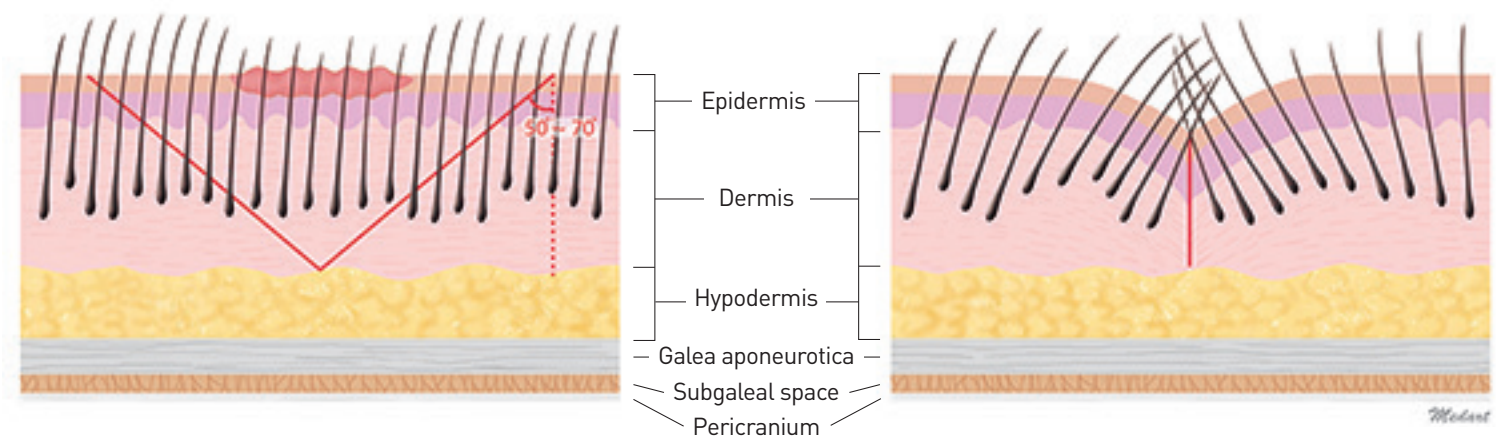

Fig. 1. An incision angle of $50-70^{\circ}$ preserved more hair follicles per skin unit. The surface irregularity and scar margin were hardly visible at 6 months.
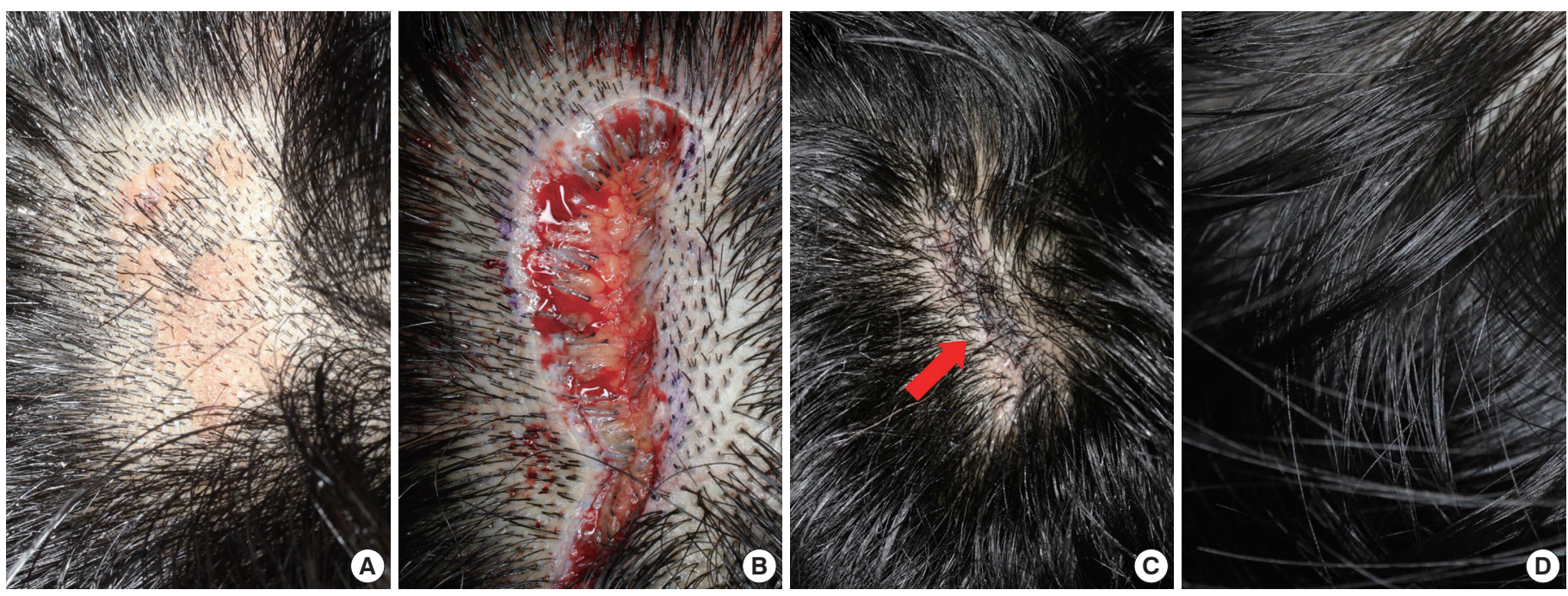

Fig. 2. Case \#2. (A) An 11-year-old boy with a $3.0 \times 1.0 \mathrm{~cm}$ nevus sebaceous on the vertex area. (B) Cross section of the excised area. Many preserved hair follicles were noted. (C) Clinical photograph at the 2 weeks follow-up shows that the scar is nearly hidden to the naked eye by obliquely growing hair (arrow). (D) Clinical photograph at the 6 months follow-up shows that many hairs from deep hair follicles grew through the scar, making the scar area unnoticeable. 
Table 1. Patients' data

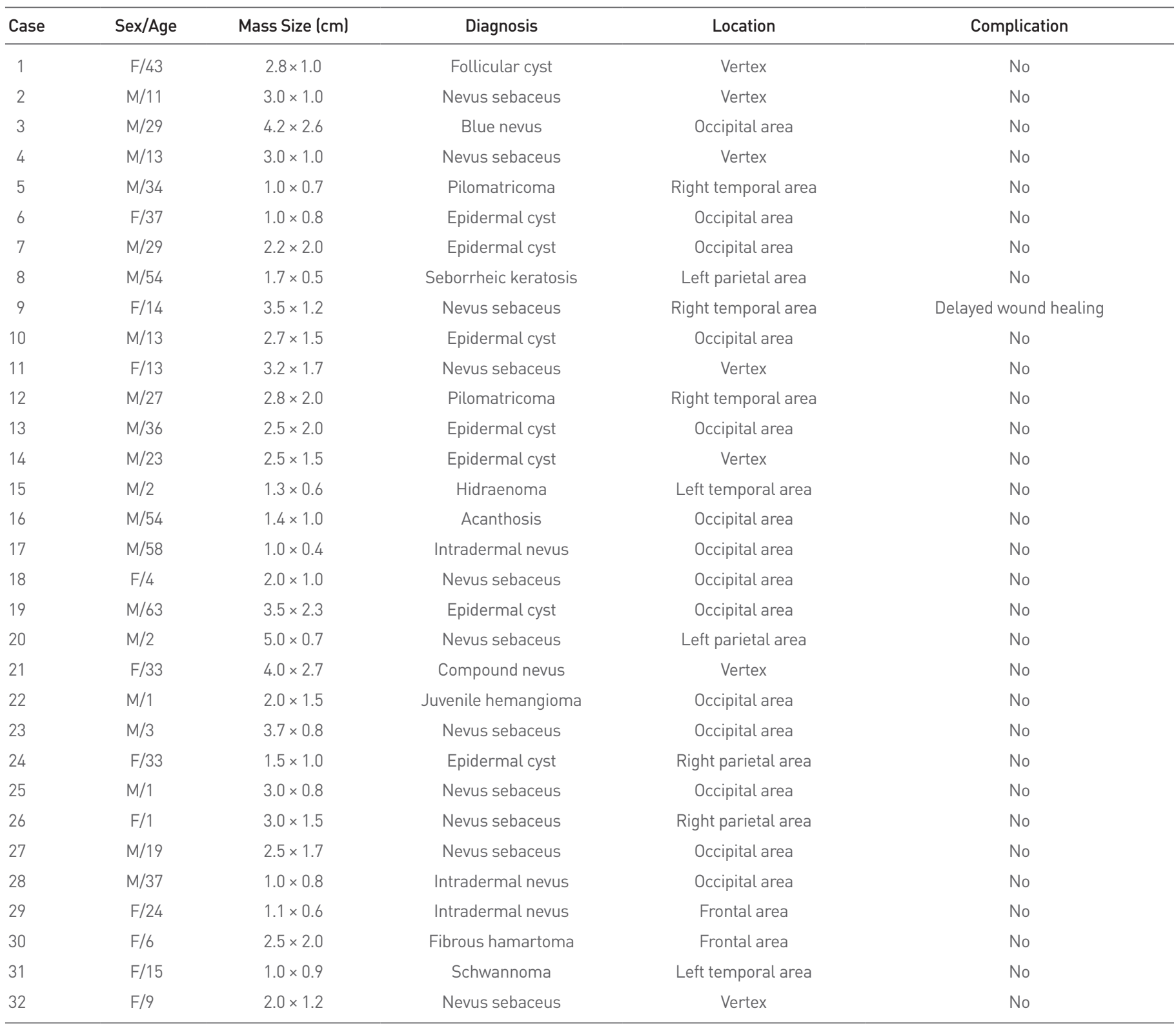

Some temporary hair loss was noticed 1 month postoperatively, but scalp hair along the scar margin regrew as time passed. Hair from the deep hair follicles grew through the scars, making the scar area unnoticeable. Observations at 3-6 months postoperatively revealed that scalp hair along the scar margin was regrowing without a large hair loss area (Fig. 2 and 3). Although some scar widening was observed, the number of hairs per unit was high enough to camouflage the scars.

One minor complication was encountered in one patient (Case \#9) who was a 14-year-old girl with a $3.5 \times 1.2 \mathrm{~cm}$ nevus sebaceous on the right temporal area. Delayed wound healing and small wound dehiscence was found. However, it was minimal and resolved spon- taneously as time passed.

\section{DISCUSSION}

Cicatricial alopecia forms a group of disorders in which the common final pathway is destruction of the hair follicle unit, which is replaced by fibrous tissue. Hair loss may occur as a primary event when the follicle is the main target of the disease process (primary cicatricial alopecia) or as a secondary event during the course of the disease occurring outside of the follicular unit (secondary cicatricial alopecia) [3]. A surgical procedure on the scalp leaves a visible scar and secondary cicatricial alopecia, which looks like a 

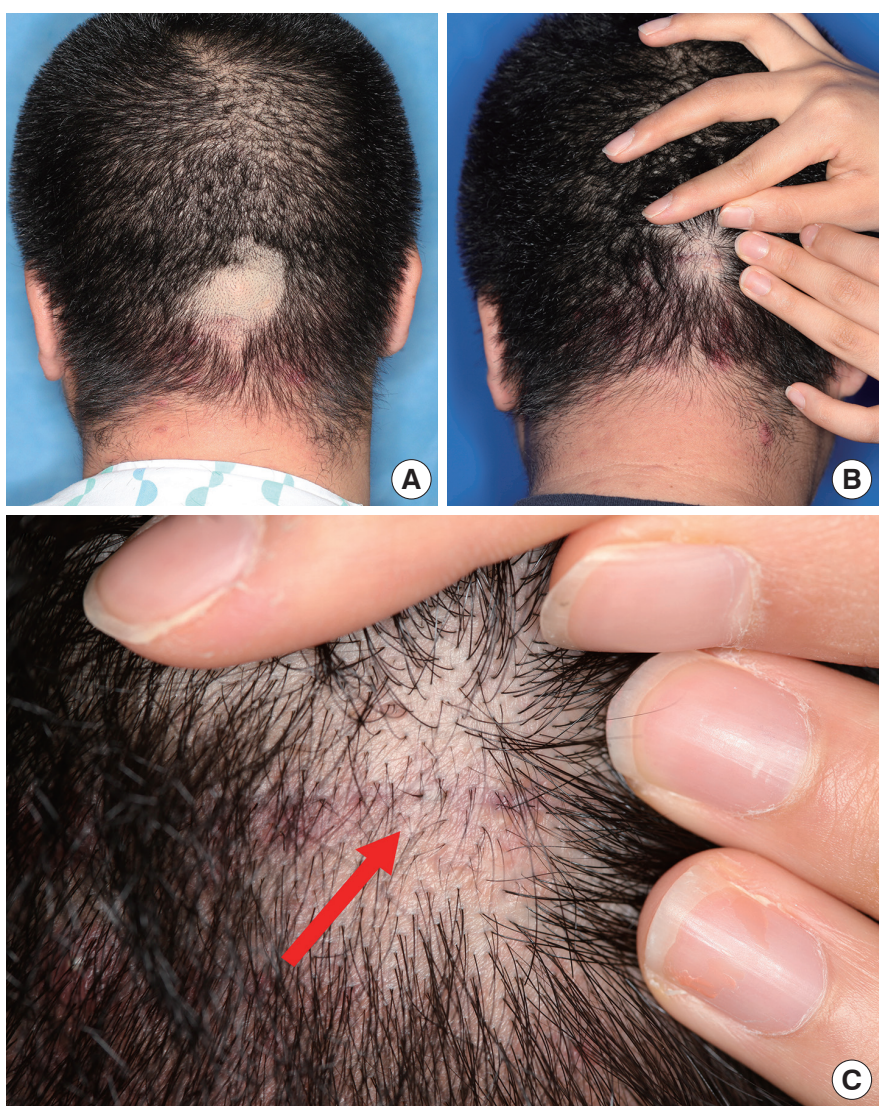

Fig. 3. Case \#7. (A) A 29-year-old man with a $2.2 \times 2.0 \mathrm{~cm}$ epidermal cyst on the occipital area. (B) Scar is nearly hidden to the naked eye by obliquely growing hair at the 3 months follow-up. (C) A magnified view reveals that the scalp hair along the scar margin regrew without a large area of hair loss (arrow).

hairless line even after optimal wound conditions. The causative factors for cicatricial alopecia are tension, use of monopolar cautery, use of key sutures with undue tension, through-and-through sutures that constrict the hair follicles and sutures left too long, as well as the injury of the hair follicles by incision [4]. Scar widening is usually the result of flap tension.

Several techniques such as the simple galeal suture, tissue expansion, skin relaxation suture, overlapping suture of the galealperiosteal flap, and anchoring of galeal flap to pericranial flaps have been recommended to avoid tension and to prevent scar widening [5]. Many researchers have explored the use of trichophytic techniques and its variations, which rely on beveling the hair follicles after donor elevation for a hair graft and overlapping the edges to improve results. However, these techniques require multiple procedures and are time-consuming, which is uncomfortable to patients and operators.

All of the established techniques have advantages and disadvantages. The simple galeal suture can minimize tension but does not avoid scar widening, at least partially [6]. After galeal suturing the scalp, the skin is disrupted more widely with a beveling incision than that with a conventional incision. Tissue expansion has an advantage of the large areas of hair deficiency. However, the obvious disadvantages are multiple clinic visits, disfigurement, cost of the expander, and long-term procedures. Additionally, the number of hair follicles per unit area decreases [7]. A skin relaxation suture with wider intervals, in which the ligation is made at some distance from the scar margin, is recommended to avoid tension and to prevent atrophy of hair stem cells, but it does not avoid partial scar widening because of its early removal [8]. The overlapping suture for galeal-periosteal flaps and anchoring of the galeal flap to pericranial flaps minimizes tension and stabilizes skin closure more than a simple galeal suture [4]. However, these techniques cannot be performed in the temporal region because the galea (the temporoparietal fascia) is placed over the temporalis muscle and its fascia and is not attached to the periosteum. Inserting dermal grafts allows the hair to grow through the scar. However, this procedure requires increased time because of its complexity [9]. Patients are happy with their result when these techniques are used, but are less than satisfied concerning the time and expense of the procedure.

We distinguish our procedure from another trichophytic closure procedure as our procedure increases tilting to an angle of $50-70^{\circ}$ to the skin surface and is simpler. The number of hair follicles per unit increases to a proper value by increasing the beveling angle, which produces a satisfactory result for both the patient and medical team. The number of hair follicles per unit decreases by decreasing the beveling angle to $<50^{\circ}$ to the skin surface, and is insufficient to camouflage the scar with the hairs. Increasing the beveling angle to $>70^{\circ}$ to the skin surface increases the number of hair follicles, but the excision area becomes wider and skin tension is higher which leads to a broad scar. Furthermore, our method requires only a single operation, which is very easy, rapid, and simple to perform.

However, the immediate postoperative skin surface is not flat, although this resolves with time. Moreover, scar widening was observed. The simplified V-shape incision technique cannot prevent scar widening, but the hairs through and around the scars are sufficient in number to camouflage the widen scars. Another unfavorable result concerns the unnatural angulation of the follicles at the scar site. These were minor concerns and did not influence patient satisfaction with the outcome.

In conclusion, despite several limitations of our study, such as the relatively short follow-up period, unnatural angulation of hair follicles, temporary surface irregularities, and use for only small sized masses on the subcutaneous fat layer, our method was very useful for scalp operations with improved scar appearance results.

\section{REFERENCES}

1. Frechet P. Donor harvesting with invisible scars. Hair Transplant Fo- 
rum Int 2005;15:119-20.

2. Marzola M. Trichophytic closure of the donor area. Hair Transplant Forum Int 2005;15:113-6.

3. Rongioletti F, Christana K. Cicatricial (Scarring) Alopecias: an overview of pathogenesis, classification, diagnosis, and treatment. Am J Clin Dermatol 2012;13:247-60.

4. Ramirez OM. The anchor subperiosteal forehead lift. Plast Reconstr Surg 1995;95:993-1003.

5. Seery GE. Anchor scalp reduction. Dermatol Surg 1996;22:1009-13.

6. Camirand A, Doucet J. A comparison between parallel hairline inci- sions and perpendicular incisions when performing a face lift. Plast Reconstr Surg 1997;99:10-5.

7. Roberts AH, Dickson WA, Dickson MG, et al. The correction of scalp hair defects by tissue expansion. Ann R Coll Surg Engl 1986;68:89-92.

8. Inaba Y, Inaba M. Prevention and treatment of linear scar formation in the scalp: basic principles of the mechanism of scar formation. Aesthetic Plast Surg 1995;19:369-78.

9. Nataf J, Elbaz JS, Pollet J. Critical study of scalp transplantations and proposal of an approach. Ann Chir Plast Esthet 1976;21:199-206. 